\title{
Geothermal Resource Exploration in Magmatic Rock Areas Using a Comprehensive Geophysical Method
}

\author{
Jie Zhu $\mathbb{D}^{1},{ }^{1,2}$ Sheng Jin $\mathbb{D}^{1},{ }^{1}$ Yang Yang ${ }^{(D)},{ }^{3}$ and Tianyu Zhang ${ }^{3}$ \\ ${ }^{1}$ School of Geophysics and Information Technology, China University of Geosciences, Beijing, China \\ ${ }^{2}$ Applied Geology Research Center of China Geological Survey, Chengdu, Sichuan, China \\ ${ }^{3}$ College of Earth Sciences, Guilin University of Technology, Guilin, Guangxi, China \\ Correspondence should be addressed to Sheng Jin; 1993010830@cugb.edu.cn and Yang Yang; sxwcyyy@163.com
}

Received 30 September 2021; Accepted 11 January 2022; Published 28 January 2022

Academic Editor: Martina Zucchi

Copyright ( 92022 Jie Zhu et al. This is an open access article distributed under the Creative Commons Attribution License, which permits unrestricted use, distribution, and reproduction in any medium, provided the original work is properly cited.

\begin{abstract}
Geothermal resources have significant development and usage potential. It is critical to conduct geological investigation of geothermal resources prior to mining, so as to deepen our knowledge and comprehension of geothermal resources. Ground water is heated by magmatic rocks and geothermal resources can be created in magmatic rock areas. However, their communication is weak, and the depth of burial is typically great. It is difficult for traditional geophysical methods, such as induced polarization method, to achieve useful exploration depths, and they have low accuracy. In this article, a comprehensive geophysical method, based on the controlled source audio-frequency magnetotelluric method (CSAMT) and transient electromagnetic method (TEM), is applied to geothermal exploration in a magmatic rock area. This method compensates for the shortcomings of a single method and achieves a good exploration effect, thereby providing a reliable geological foundation for further development and utilization of geothermal resources.
\end{abstract}

\section{Introduction}

In China, there are enormous reservoirs of geothermal resources, most of which are reserved in the form of liquiddominant systems and mainly distributed throughout southern Tibet, western Sichuan, western Xinjiang, western Yunnan, and Taiwan (Figure 1) [1]. They have a total thermal value of $3.06 \times 1,018 \mathrm{kWh}$ per year, accounting for approximately $8 \%$ of the global geothermal reservoirs [2]. However, at present, China's exploited geothermal resources account for less than $5 \%$ of reserves, and continued development and exploitation of geothermal resources bears promising potential [3]. The buried depth of geothermal resources is typically enormous, with most exceeding $2,000 \mathrm{~m}$, and the hazards involved in exploiting are significant. To obtain a better knowledge of geothermal resources, it is important to undertake a geological survey prior to exploiting, so as to determine their depth, position, and reserves. In the exploration of geothermal resources, geophysical methods have been shown to be effective $[4,5]$.
At present, a variety of geophysical methods have been successfully applied in geothermal exploration. Soengkono et al. [6] used the magnetic method to explore in potential geothermal areas and successfully delineated the scope of geothermal resources in 1992. Liu and Zhang [7] used the microgravitational method to conduct geothermal exploration and were successful in Xianning, Hubei, China, in 1994. Xu et al. [8] analyzed the application effects of the gravitational method, the magnetic method, the micromotion method, the controlled source audio-frequency magnetotelluric method (CSAMT), the magnetotelluric method (MT), and other methods in the sedimentary basins of northern China in 2007. Guo et al. [9] applied the remote-sensing and the electromagnetic methods to the convective geothermal field in Qinghai, China, in 2012. The remote sensing was used to divide the geological structure and determine the thermal target area, and the electromagnetic methods were used to determine the location, depth, and tendency of fault structures. 


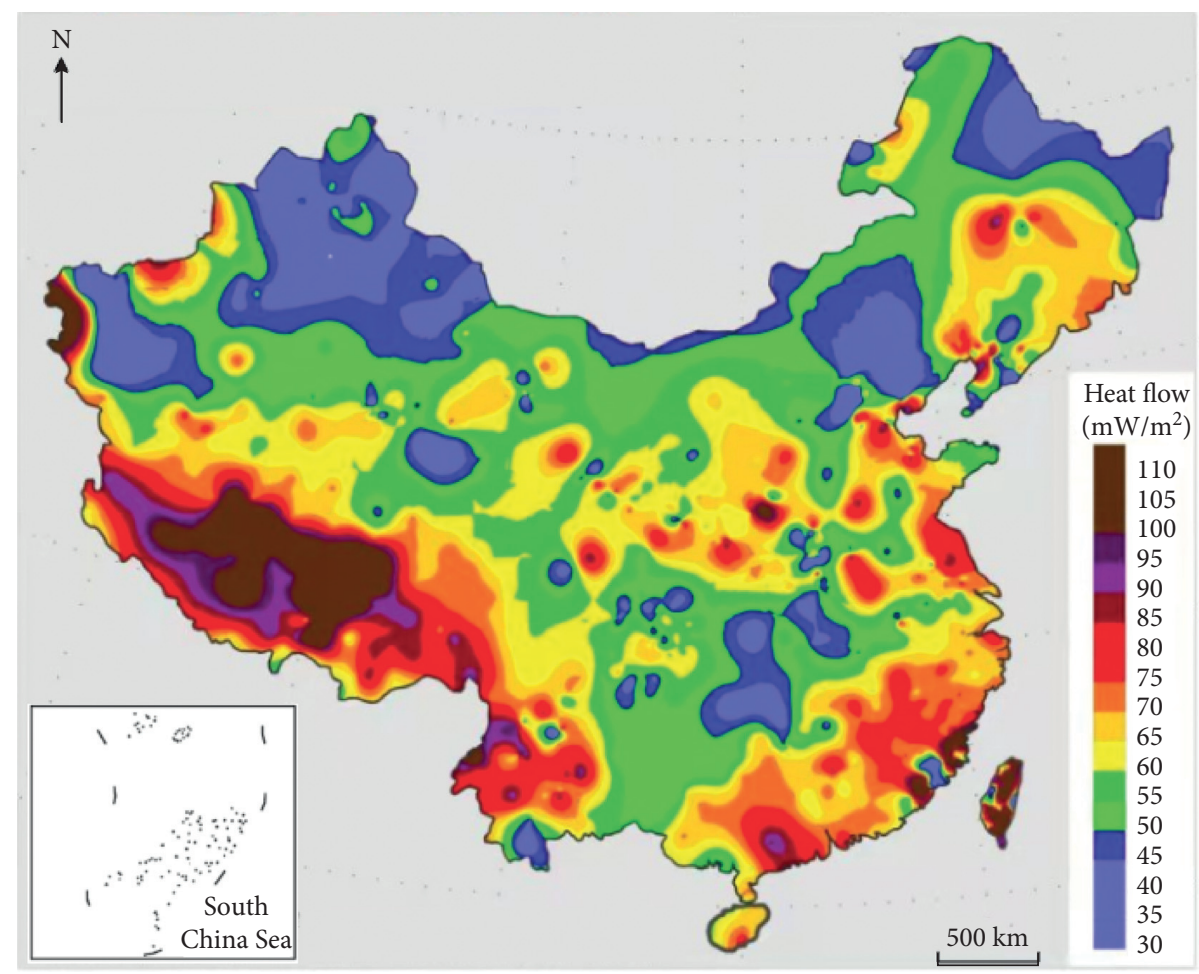

Figure 1: Heat flow map of China (cited from Hu et al., 2011 [1]).

Among geophysical methods, electromagnetic methods have achieved relatively strong results in geothermal exploration, including MT, which is commonly used in deep geothermal exploration, along with the audio-frequency magnetotelluric method (AMT), CSAMT, and the transient electromagnetic method (TEM) [10-15]. However, geological conditions and human interference vary greatly across exploration areas, making it difficult to use electromagnetic methods to explore targets. Furthermore, due to the complex geological units involved in geothermal, the exploration depth required is relatively large, and simultaneous exploration is difficult. As a result, under the assumption that all methods are capable of achieving a certain exploration effect, it is critical to conduct geothermal exploration using multiple methods in order to fully exploit the advantages of each one, which bears significant theoretical and practical implications.

In this article, we first analyzed the geothermal system model of the magmatic rock area, establishing the geophysical basis for the electromagnetic method to explore geothermal resources. Next, we analyzed the method characteristics of CSAMT and TEM, and compared the advantages and limitations of them from the form of the transmitting source. After this, we explained the resolution for different targets, as well as the complementarity of CSAMT and TEM. The comprehensive electromagnetic method, with CSAMT as the mainstay and TEM as a supplement, was applied to the geothermal exploration engineering practice in Huairen County, Shanxi Province, China.

The comprehensive electromagnetic method based on CSAMT and supplemented by TEM has been shown in theoretical and practical analyses to effectively compensate for the shortcomings of a single method and has achieved a relatively ideal geothermal exploration result in magmatic areas.

\section{Theoretical Principle of Geothermal Systems}

The "heat" contained in the Earth's interior is referred to as geothermal energy. This heat is radiated outwards from the Earth's core until it reaches the permeable and broken stratum near the surface. When this geothermal energy heats water, the hot water or steam is stored in a permeable and porous rock surrounded by an impermeable layer, thereby forming a geothermal reservoir or geothermal system [16]. The circulating water in a geothermal system is typically drawn from the atmosphere and serves as "replenishment" for the underground geothermal fluid. Atmospheric precipitation can completely or partially replace the liquid that flows out of the reservoir via spring water or boreholes. After the thermal energy in the magma heats the groundwater, the thermal energy is carried to the shallow geological unit with high porosity through the circulation of water and then flows out in the form of hot springs [17, 18]. Figure 2 shows a simplified form of an ideal geothermal system.

The geothermal system presents significantly different distribution laws under different geological blocks and geological conditions, and the geothermal system is the result of the combined action of several geological elements. For a typical geothermal system in a magmatic rock region, the four major elements, thermal source, thermal reservoir, thermal cap, and migration channel, are indispensable. The 


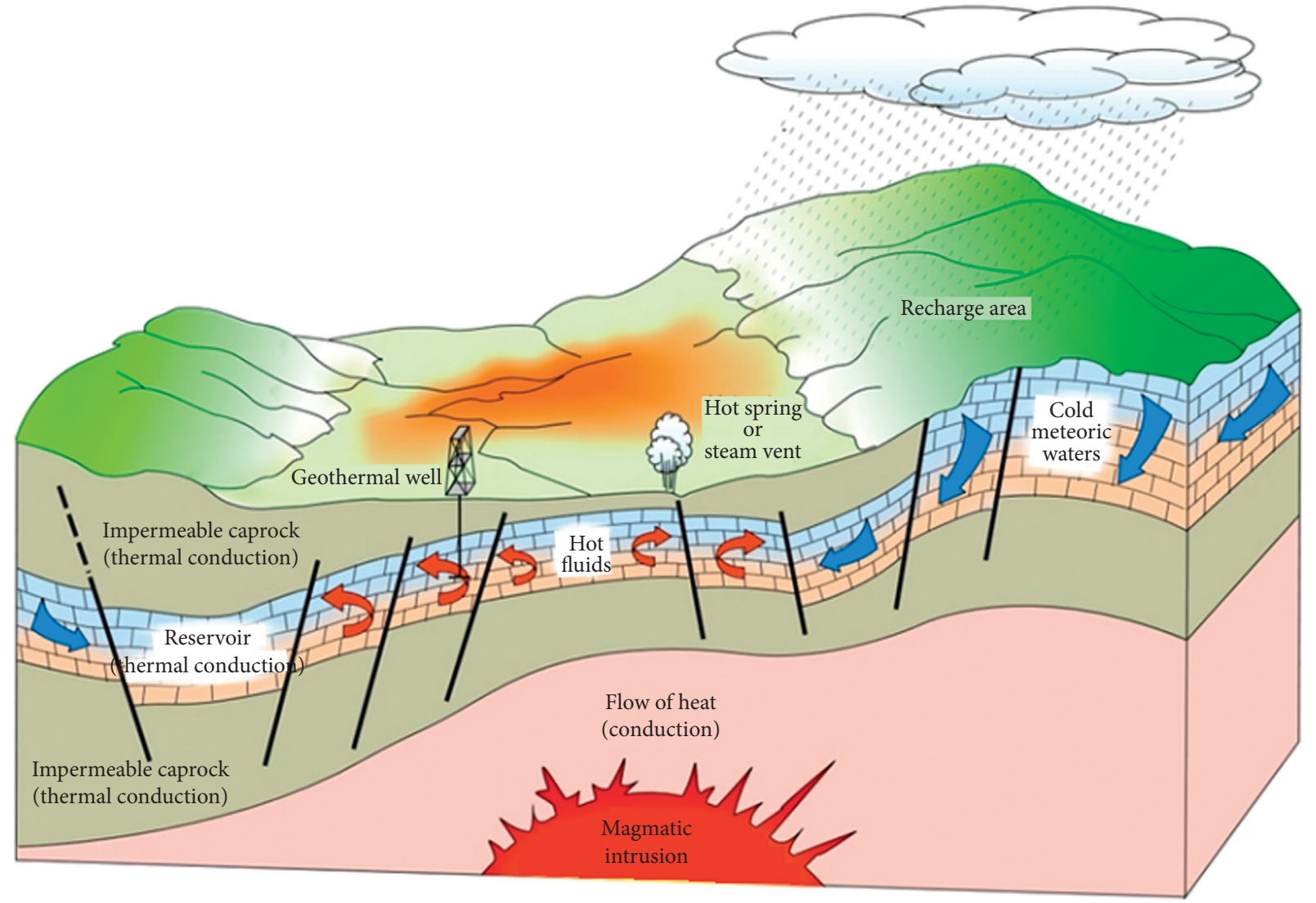

Figure 2: Schematic diagram of an ideal geothermal system (cited from Huenges, 2016 [19]).

main factor closely related to the electromagnetic method is electrical resistivity, which is directly affected by other factors such as the degree of formation water content, waterbearing mineralization, rock lithology, porosity, and heat storage structure temperature. Among them, the most important factor is the lithology of the rock. In general, metamorphic and magmatic rocks have higher resistivity, whereas sedimentary rocks have lower resistivity. At the same time, the presence of the liquid medium in the rock will also significantly alter its resistivity. The difference in the resistivity of formation rocks is the physical basis of electromagnetic exploration [20].

\subsection{Elements of the Geothermal System}

2.1.1. Thermal Reservoir. Geothermal fluids are stored in geological structures, which are called geothermal reservoirs, and these geological structures are generally rock formations exhibiting great porosity and permeability. In the available geothermal resources (hydrothermal systems), geothermal fluids can possess different sources, such as magmatic, metamorphic, meteoric, or a combination of these. In this study, the main heat source of the geothermal fluid is magmatic heat. At this time, the basic structure of the reservoir is deep bedrock. Compared with the shallow stratum, the deep bedrock as a geothermal reservoir generally has high resistivity, which is the main target for electromagnetic exploration [21].
2.1.2. Thermal Cap. The thermal cap layer is an impermeable or weakly permeable geological structure, which insulates the thermal reservoir from exchanging energy with the outside world. It mainly plays the role of heat preservation and heat insulation in the geothermal system. The thermal cap layer not only bears the function of insulating heat exchange but can also prevent the loss of geothermal fluid. Therefore, in general, the thermal cap layer is dense. In magmatic rock regions, common thermal caprocks include Quaternary alluvial beds with silt, peat soil, and loam, as well as granite and residual soil, all of which are in direct contact with thermal reservoirs. When the continuity of the thermal capping layer is poor and the sealing of the thermal storage is insufficient, then the geothermal fluid will overflow in the form of hot springs at its missing parts. Due to the shallow burial depth and the susceptibility to weathering, the resistivity of the thermal cap layer is lower than that of the thermal reservoir [22].

2.1.3. Thermal Source. The heat source of the geothermal system originates from the deep parts of the Earth, mainly including magma waste heat, radioactive element transformation heat, and upper mantle conduction heat [23]. Different thermal fluids have different heat sources. In hightemperature or magmatic rock regions, the thermal fluid mainly originates from the magma waste heat; while in medium- and low-temperature regions, the thermal fluid mainly originates from the deep asthenosphere, which is 
tangent to the deep lithosphere through the large deep fault. The deep mantle material of the Earth flows upward along the migration channel, creating the channel between the thermal reservoir and deep heat source. In this process, geothermal water relies on deep circulation and geothermal gradients to obtain heat and store it in the thermal reservoir [24]. Therefore, the electrical characteristics of the heat source are consistent with the electrical characteristics of the thermal reservoir, generally showing high resistance.

2.1.4. Migration Channel of Geothermal Fluid. The migration channels of geothermal fluid are mainly faults. Here, "faults" specifically refer to the fault parts in the geothermal system as migration channels, rather than the "faults" in the broad sense of geology. These faults are generally in large scales, with good water conduction and thermal conductivity, to meet the space requirements of fluid migration. At the same time, in many cases, the closure of the boundary of the migration channel is superior, which can block the largescale loss of fluid during the migration process [25-33]. Compared with the thermal caprock, the migration channel of geothermal fluid has strong water richness and low resistivity; thus, it is also the main target for geothermal resource exploration in magmatic rock regions.

In summary, thermal reservoirs and fluid migration channels are the main targets for electromagnetic exploration methods to explore geothermal resources in magmatic rock regions. Compared with surrounding rocks, thermal reservoirs have high resistivity, whereas migration channels have low resistivity. In magmatic rock regions, the resistivity anomaly as the exploration target is often a comprehensive reflection of the thermal storage stratum and fault structure, rather than the thermal storage structure itself. Therefore, for geothermal exploration in magmatic rock regions, it is particularly critical to explore the location, shape, and depth of fault structures and thermal reservoirs. The main purpose of exploration is often to determine whether there are geothermal-related faults in magmatic rock regions [34, 35].

\subsection{Geophysical Characteristics of Geothermal Resources in} Magmatic Rock Areas. The conductivity of different rock formations differs. Due to the ancient diagenesis age, most magmatic rock areas remain compact and complete after longterm metamorphism, and cracks do not form. The resistivity is generally consistent in the horizontal direction and gradually increases with increasing depth in the vertical direction, making it unsuitable for geothermal resource exploration [36].

In some magmatic rock areas, the pore water of the overlying loose layer can flow into the deep magmatic rock and the fissures, after having received vertical infiltration replenishment of atmospheric precipitation and intermittent leakage replenishment of surface water. Thermal storage aquifers are underground hot water formations, which store hot water in magmatic rock formations. The resistivity of eruptive rocks (such as basalt and rhyolite) is generally low $(20-500 \Omega \mathrm{m})$, which is lower than that of granite (greater than $500 \Omega \mathrm{m}$ ) (Table 1), but the resistivity of magmatic rocks is generally greater than that of sedimentary ones. As a
TABLe 1: Statistical of rock resistivity in the magmatic area.

\begin{tabular}{lccc}
\hline Lithology & Resistivity $(\Omega \cdot \mathrm{m})$ & Lithology & Resistivity $(\Omega \cdot \mathrm{m})$ \\
\hline Sand & $60-100$ & Conglomerate & $50-200$ \\
Silty clay & $14-25$ & Limestone & $100-300$ \\
Basalt & $20-500$ & Magmatic rock & $>1000$ \\
Granite & $500-2000$ & & \\
\hline
\end{tabular}

result, geophysical prospecting methods can measure the difference in resistivity values between magmatic rocks and sedimentary surrounding rocks, so as to determine the location of magmatic rocks, and then search for geothermal resources [37].

On the other hand, the resistivity value will change in magmatic areas where faults form. The fault's activity may cause a fracture zone near the fault, and the fault may result in a hydraulic connection between the upper and lower strata, in turn forming a low-resistivity anomaly area near the fault which is close to the fault's dip angle. These lowresistivity anomalies exist in resistivity. The cross-sectional view is generally relatively steep. It can reflect the existence of faults and then search for geothermal resources by seeking such a steep anomaly with low resistivity $[38,39]$.

\section{Methodology}

3.1. Controlled Source Audio-Frequency Magnetotelluric Method. The controlled source audio-frequency magnetotelluric method (CSAMT) is a frequency-domain artificial source electromagnetic technology developed on the basis of the magnetotelluric method (MT). Some of its advantages are as follows:

(1) Large exploration depth: In CSAMT work, parameters such as the transmission frequency range and transmission and reception distance can be set according to the geoelectric characteristics of each work area. Compared with the loop-source TEM, when a lower emission frequency is used, the exploration depth is greater, up to $2 \mathrm{~km}$ or more.

(2) Strong ability to penetrate the high-resistivity shielding layer: This method receives perpendicular electric and magnetic fields at the same time, the electromagnetic signal is perpendicular to the ground in the receiving area, and the observation and calculation of the electromagnetic component have been normalized. Therefore, it can effectively penetrate the high-resistivity cap layer on the ground.

(3) Larger transmitting power and strong anti-interference ability: This method adopts an artificially controllable transmitting source, with high transmitting power (transmitting power up to $30 \mathrm{~kW}$ ), capable of precise frequency division, excellent stability, and high-order superposition. In addition, it can realize the function of suppressing the interference of ground electricity.

(4) High effectiveness for the exploration of high-resistivity basement formations: Where the 
exploration depth is large, generally in high-resistivity basement formations, at this time, CSAMT uses a lower operating frequency (less than $1 \mathrm{~Hz}$ ) as the transmitting signal, and the signal attenuation in this frequency band is slower. Compared with other electromagnetic exploration methods, CSAMT can still effectively explore deep high-resistivity formations, thus making it more effective in exploring relatively high-resistivity basement formations.

\subsubsection{Limitations of CSAMT}

(1) Affected by the undulations of the surface or shallow terrain, the apparent resistivity distortion of the shallow part is caused, that is, the "static effect": the electrical properties near the surface are not horizontally inconsistent or caused by uneven terrain, which will measure all electric fields. The accumulation of charges on the surface of the inhomogeneous body will cause errors in the electric field data, and the apparent resistivity will also change, thereby forming a "static effect."

(2) The longitudinal resolution of the shallow formations is relatively reduced: CSAMT has a large transmission frequency range (from $0.125 \mathrm{~Hz}$ to nearly $10,000 \mathrm{~Hz}$ ). In order to consider the effect of deep exploration, the frequency interval in the high frequency range is set to be relatively large. If it is larger, then the longitudinal distance between the shallow surveying points corresponding to the high frequency is also larger, and in turn, the longitudinal resolution of the shallow stratum is relatively reduced.

(3) The lateral resolution is limited, and the ability to explore faults and other structures is somewhat weak: in principle, the lateral resolution of CSAMT is approximately equal to the size of the receiving electric dipole. However, the geological environment of each work area is complex, and the stratum is not a homogeneous or layered medium, and thus, the lateral resolution of CSAMT is a comprehensive result of a variety of geological factors. Particularly in the case of sloping strata and complex surface conditions, the lateral resolution of CSAMT sometimes exceeds $200 \mathrm{~m}$, which reduces the ability to explore faults and other structures.

(4) In actual construction, electrode pits must be arranged during signal transmission. It is necessary to manually dig the soil and lay aluminum foil on the bottom and side walls of the electrode pits to reduce the surface resistivity. The construction steps are quite complex, and the requirements for transmitting power and observation area are relatively high.

3.2. Transient Electromagnetic Method. The transient electromagnetic method (TEM) is a time-domain artificial source electromagnetic method. TEM conducts observations within a short period of time after the primary field has been turned off and the secondary has yet to be started. The data are not directly related to the primary field and are separated from the primary field in time, which is known as separability in time. Calculated according to the sampling time, the secondary signal received at a certain time node is caused by the inconsistency of various exploration depths. After comprehensive analysis of the collected secondary signal and resistivity, the geological information of each depth can be obtained, that is, separability in space [40-43].

Based on these two separabilities, TEM has the following advantages:

(1) Minimally affected by terrain: In high-resistivity surrounding rock areas, there will be basically no false anomalies caused by the effect of terrain; in lowresistivity surrounding rock areas, the secondary field signal is used for data measurement; thus, it can be used for early identification of the impact of terrain [44].

(2) Simplified construction method and high efficiency: The data collection process is simple, and there are no strict requirements regarding the position of the coil and receiving distance; thus, the field construction is easy to carry out. During the construction, the profile and depth sounding work are carried out at the same time, and the work efficiency is high.

When collecting data and information, the receiving system is close to the vertical position of the target body; thus, it can be closely coupled to improve the surveying ability of the target body $[45,46]$.

(3) High vertical resolution at the shallow surface, and strong ability to explore low-resistivity formations on the surface: TEM is a time-domain electromagnetic exploration method. The time window for data observation (sampling time $\boldsymbol{t}_{\text {off }}$ ) can be controlled by controlling the sampling time of the early traces to improve the longitudinal resolution of the shallow strata.

TEM observes a pure secondary field, known as "pure anomaly," and the secondary field response of low-resistivity bodies is quite obvious. Therefore, by observing the secondary field signals of the early traces, low-resistivity formations on the surface can be effectively explored [47].

(4) High lateral resolution and sensitive reflection of low-resistivity bodies such as water-conducting fault structures: In TEM measurement, the secondary field potential of multiple measurement channels can be arranged according to the measurement points, namely, "multiple surveying channels." In the "multichannel map" and "resistivity cross section map," from the distribution of the secondary field potential and resistivity along the surveying points, we can clearly observe the electrical characteristics of the formation in the horizontal direction. We can then alter the situation to obtain a higher horizontal resolution. 
When there are low-resistivity bodies such as waterconducting fault structures, the secondary field decays slowly and the apparent resistivity is low, that is, the characteristics of "high potential and low resistance," which can have a relatively significant exploration effect on lowresistivity structures [48].

\subsubsection{Limitations of TEM}

(1) Poor anti-interference ability: TEM is susceptible to interference from noise currents, especially highvoltage lines. At present, there is no effective method to suppress noise. The only way to reduce interference is to increase the number of superpositions and increase the emission current;

(2) Small exploration depth: The TEM exploration depth $\mathrm{H}$ in a homogeneous medium can be expressed as follows [49]:

$$
H=0.55\left[\frac{M \rho}{R_{m} N}\right],
$$

where $M$ represents the magnetic moment of the transmitter coil, $\rho$ represents the average resistivity value of the exploration depth $H$, and $R_{m N}$ represents the minimum signal level that the transient electromagnetic instrument can distinguish. It can be seen from this formula that the exploration depth $H$ is positively correlated with $M$ and $\rho$, and when $M$ or $\rho$ increases twice, the exploration depth $H$ only increases by $15 \%$. In the actual construction process, in general, the effective area $S$ of the launch loop can only be increased by increasing the side length of the launch loop, after which the magnetic moment $M$ of the launch coil is increased to increase the exploration depth, which leads to a certain extent reduced resolution and construction efficiency;

(3) Large deep data error: Due to the fact that the TEM observes the secondary field, when the exploration depth is large, the secondary field signal of the late track is already weak, and the V/I value of the secondary field attenuates below $1 \mathrm{mV} / \mathrm{A}$. The acquisition accuracy is inevitably reduced, causing a large error in the calculated resistivity;

(4) Blind zone: In the same manner, as the TEM observes the secondary field, in order to avoid the influence of the primary field, the sampling time $\boldsymbol{t}_{\mathrm{off}}$ cannot be started too early, which leads to a blind zone appearing in the shallowest part of the surface of the TEM. The expression of the blind zone depth is as follows [50]:

$$
h \approx 28 \sqrt{\rho t}
$$

where $h$ represents the blind zone depth of the homogeneous medium, $\rho$ represents the resistivity value $(\Omega \cdot \mathrm{m})$ below the surveying point, and $t$ represents the time (ms) for the initial observation of the effective transient field, which is the turnoff time $t_{\text {off. }}$

3.3. Comparison of Ability to Explore Low-Resistivity Target between CSAMT and TEM. CSAMT is a frequency-domain method, whereas TEM is a time-domain method. The difference between the two is directly reflected in the emission waveform. The difference in emission waveform causes CSAMT and TEM to differ in the means of data observation. CSAMT transmits and receives through a single frequency point in the frequency domain to obtain a response signal. TEM transmits wide-band signals in the time domain and collects wide-band responses, and signals at all frequency points can be obtained with a single transmission. From the perspective of data collection efficiency, TEM is superior to CSAMT. However, in the CSAMT transmission mode, the energy of a single frequency point in the source signal is greater than the energy of a single frequency point in the TEM transmission mode; thus, CSAMT can obtain a higher signal-to-noise ratio at each frequency point than TEM.

In terms of resolution, the difference between CSAMT and TEM is mainly caused by the different types of emission sources of the two methods. The return line sources TEM and CSAMT use ungrounded return lines and grounded long wires to send signals to the ground, respectively, thereby resulting in essential differences in the electromagnetic fields used by the two methods. Under the excitation of a long ground wire, due to the existence of the ground term, there will not only be a horizontal current $\left(\boldsymbol{E}_{1}\right)$ induced by induction in the Earth but also be a vertical induced current $\left(E_{2}\right)$. Consequently, the electromagnetic field excited by the long wire source contains the component of the TE mode and can also produce the TM mode. In addition, the nongrounded loop device can only generate horizontal current $\left(\boldsymbol{E}_{1}\right)$; thus, there is only the electromagnetic field of TE mode (Figure 3). Due to the fact that the TE mode is sensitive to low-resistivity objects but not to high-resistivity objects, the loop-source transient electromagnetic exploration method possesses poor resolution capabilities for high-resistivity targets.

On the other hand, CSAMT measures electric and magnetic field components, whereas TEM measures magnetic field components. When the target fault of the local thermal system is covered by a shallow low-resistivity layer, the abnormal response generated by the layer boundary charge accumulation will submerge the low-resistivity target fault, making the target fault difficult to distinguish. In fact, the influence of the layer boundary charge accumulation on the electric field is much greater than that on the magnetic field. On this basis, the loop-source TEM can produce a superior resolution of target faults in this environment. In actual exploration, since the complexity of underground media is far greater than that of geophysical analysis and modeling, comprehensive exploration by applying both CSAMT and TEM can undoubtedly provide more information and evidence for distinguishing target faults and other geological bodies. 

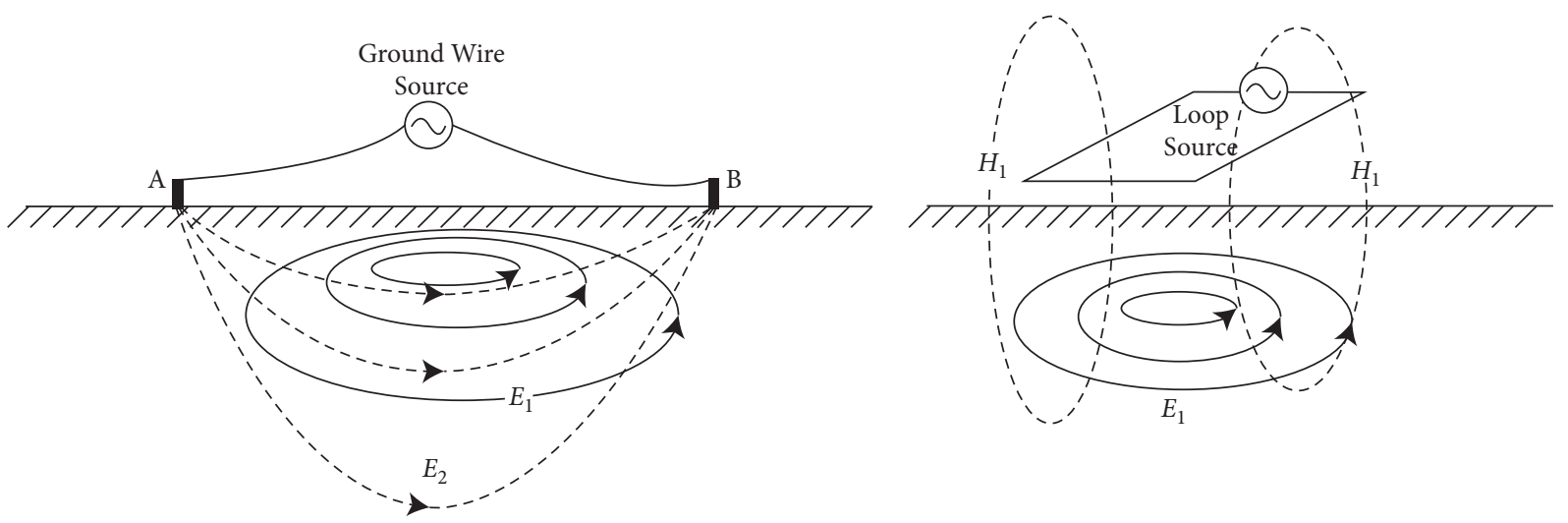

Figure 3: Comparison of the underground current distribution stimulated by the ground wire source and the loop source in a uniform half space; the left diagram is simulated by using a ground wire source, whereas the right diagram is simulated by using a loop source. (E) 1 represents horizontal current, (E)2 represents vertical induced current, and (H)1 represents vertical magnetic field.

\section{Experimental Analysis}

In order to verify the effects of the comprehensive geophysical methods on geothermal resource exploration in magmatic rock areas, a geothermal resource exploration with comprehensive geophysical methods was carried out at a location in Huairen County, Shanxi Province, China.

This area is located in the uplift belt on the west side of the central part of the Sanggan River New Rift in the Datong Basin. It straddles the Huairen Sag and Huanghualiang Sag uplift from west to east. Ordovician (O), Carboniferous (C), Permian $(\mathbf{P})$, and Cenozoic $(\mathbf{Q}+\mathbf{N})$ strata occur, with Archean (Ar) granite and Late Tertiary $(\mathbf{T})$ basalt intrusions occurring locally, among which basalt and deep magmatic rocks of the Wutai Group are strong underground thermal storage sources of water.

This area straddles two structural units, the Huairen Graben and Huangliang Horst, which are secondary structures of the New Rift of the Sanggan River. A series of NE-strike fault structures occur in the area. The development of fault structures provides a superior connection channel for various underground aquifers (Figure 4).

As shown in Figure 5, four CSAMT surveying lines are arranged in the study area.

In the CSAMT exploration, the instrument used is the GDP32II multifunctional electrical method workstation. The transmitting pole distance $A B$ is $1,500 \mathrm{~m}$, the transmitting current is $14-16 \mathrm{~A}$, and the transmitting distance is $6 \mathrm{~km}$; the receiving point distance of Line 59 and Line 64 is $50 \mathrm{~m}$, the receiving point distance of Line 10 and Line 60 is $100 \mathrm{~m}$, and the signal frequency range is $0.125-8,192 \mathrm{~Hz}$.

In the TEM exploration, the instrument used is also the GDP32II multifunctional electrical method workstation, and the center loop device is applied for measurement. The transmitting source is a $600 \times 600 \mathrm{~m}$ single-turn loop, powered by a generator, the fundamental frequency of the transmitting source is $16 \mathrm{~Hz}$, and the transmitting current is $15 \mathrm{~A}$; the distance between the surveying points is $50 \mathrm{~m}$.

After the CSAMT exploration has been completed, a TEM surveying line is arranged near the 60 line where the
CSAMT abnormality is obvious. This is then used to perform a comparison with the CSAMT results.

4.1. Verified Situation. In the processing of the measured data, we first use GDP32II data preprocessing software to sort the collected data and remove the dead pixels. Next, we use CSAMT-2D and TEM-1D software based on the OCCAM algorithm to invert the data. The inversion results of CSAMT and TEM resistivity profiles are as follows.

In general, the morphologies of the resistivity profiles of CSAMT and TEM have some similarities, and both show that the resistivity is medium-low in the medium and shallow layers (Figure 6). In addition, the resistivity gradually increases as the depth increases, and basement magmatic rock also exhibits high-resistivity characteristics.

Comparing the exploration effects of CSAMT and TEM, the differences are as follows:

(1) TEM is superior to CSAMT in the exploration effect at shallow depths on the surface. CSAMT is affected by topography. The low-resistivity shielding interference of the Quaternary muddy sand and clay layer is relatively large, which exhibits abnormal medium resistance, and the resistivity curve presents characteristics such as distortion, which reduces the resolution of shallow layers to a certain extent. Although there is a blind zone with a depth of approximately $50 \mathrm{~m}$ in TEM, the low-resistivity shielding layer at the shallow surface undergoes less interference, and the resistivity value exhibits obvious regularity at the shallow surface, which is consistent with the actual geological characteristics.

(2) CSAMT is superior to TEM in terms of exploration effect at large depths. In the section of 4,500-10,000 m, CSAMT has superior data stratification in the deep part, while the TEM resistivity curve in this section is slightly unclear, and the TEM shows a circle of the resistivity curve in the section of $9,000-9,500 \mathrm{~m}$. As for the closed high-resistivity abnormal value, this high-resistivity abnormal value 


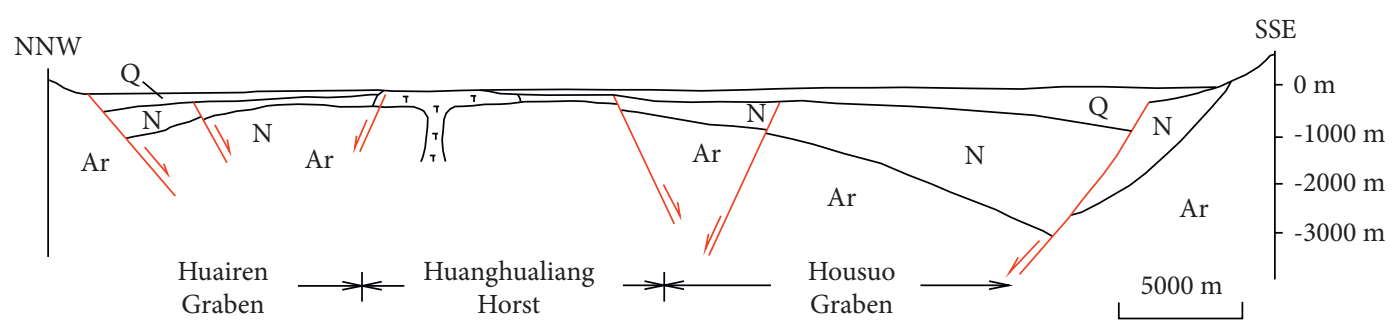

FIGURE 4: Geological section diagram of new rift of the Sanggan River, which contains Huairen Graben, Huanghualiang Horst, and Housou Graben, illustrating the geological structures of the study area. (Q) represents Quaternary strata, (N) represents Neogene strata, and (A) (r) represents Archean strata.

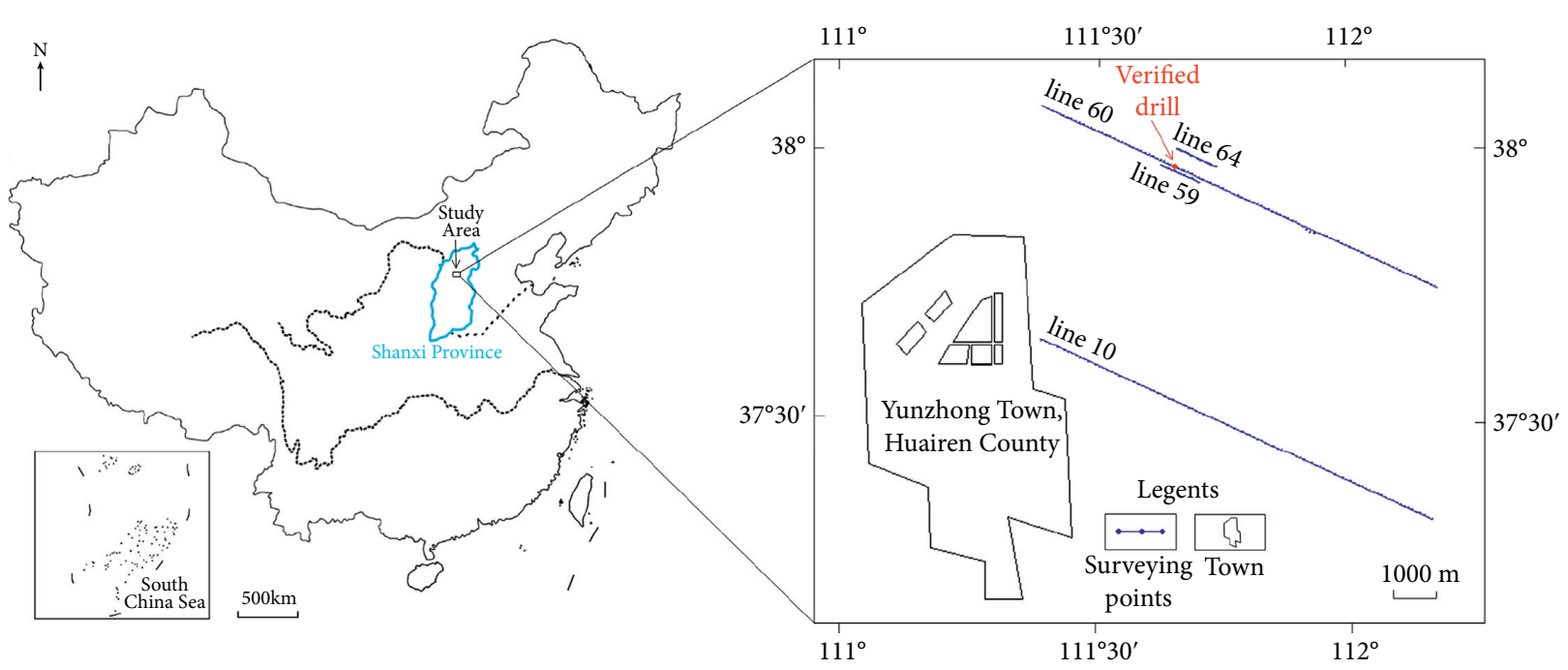

FIGURE 5: Project layout that contains the location of the study area in Shanxi Province in northern China, showing the base map of the study area.

should be a false abnormality caused by interference. The secondary field potential of the late TEM measurement track also fluctuates greatly in this section, lacking regularity, thus indicating that CSAMT has superior anti-interference ability than TEM.

(3) TEM and CSAMT have their own advantages in the exploration of water-conducting fault structures.

Due to the fact that the pure TE field mode of TEM is particularly sensitive to low-resistivity targets [51], this is more obvious in the reflection of F1 fault. CSAMT does not effectively reflect the F1 fault in the low-resistivity area in the shallow part, yet there is obvious low-resistivity anomaly on the TEM profile. It can also be seen from the TEM secondary field potential multichannel map that there is an obvious abnormal high value of the secondary field potential in this area.

The advantage of CSAMT for fault exploration is mainly reflected in the exploration of the deep highresistivity basement interruption layer. In the sections of $5,000-5,700 \mathrm{~m}$ and $9,500-10,000 \mathrm{~m}$, it is inferred that the buried depth of the Quaternary and Tertiary loose deposits is approximately $500 \mathrm{~m}$. The lower part is the basement of magmatic rock, and the resistivity reflects the characteristics of high resistance. In the CSAMT cross section map, these two sections appear as steep gradient zones, which are inferred to be F2 and F3 faults. These can more intuitively distinguish the upper and lower walls of the fault.

(4) CSAMT is superior to TEM for the exploration effect of magmatic rock basement. In the section of 5,800-9,300 m, CSAMT exhibits a very obvious highresistivity response to the magmatic rock basement, while the high-resistivity response of TEM is not obvious at this position, and it does not highlight the magmatic rock basement.

Following the completion of the geophysical prospecting construction, the drilling verification was carried out at the 3,350 surveying point. The drill encountered underground hot water at $1,610 \mathrm{~m}$, after which a pumping test was carried out. According to the results of the pumping test, the unit output of underground hot water in this area was $233 \mathrm{~m}^{3} / \mathrm{d}$. The water temperature was $58^{\circ} \mathrm{C}$. These results confirmed the occurrence of geothermal water in the faults and magmatic rock formations of the Wutai Group. 


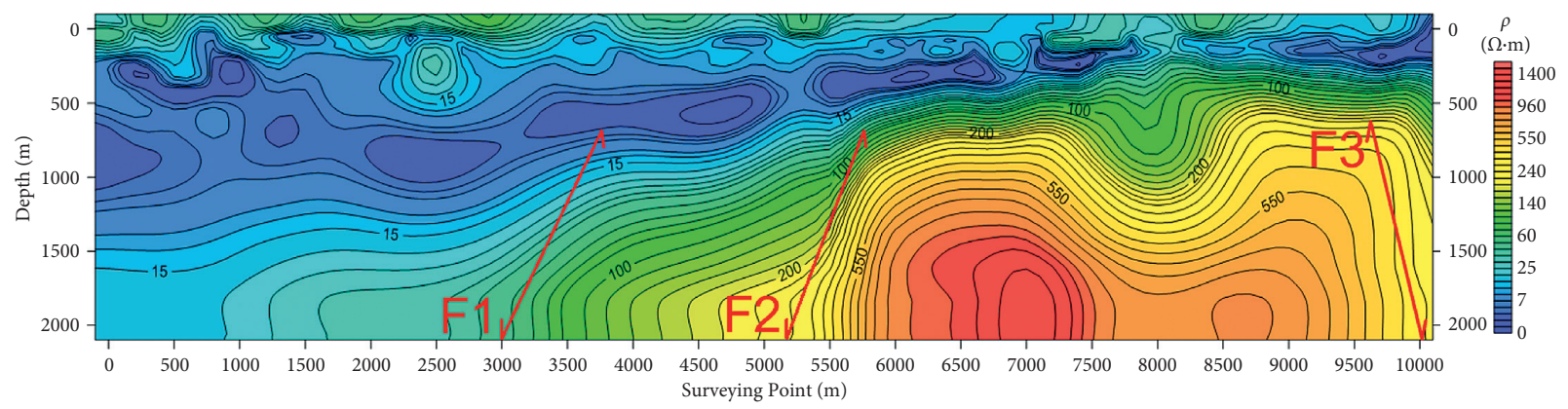

(a)

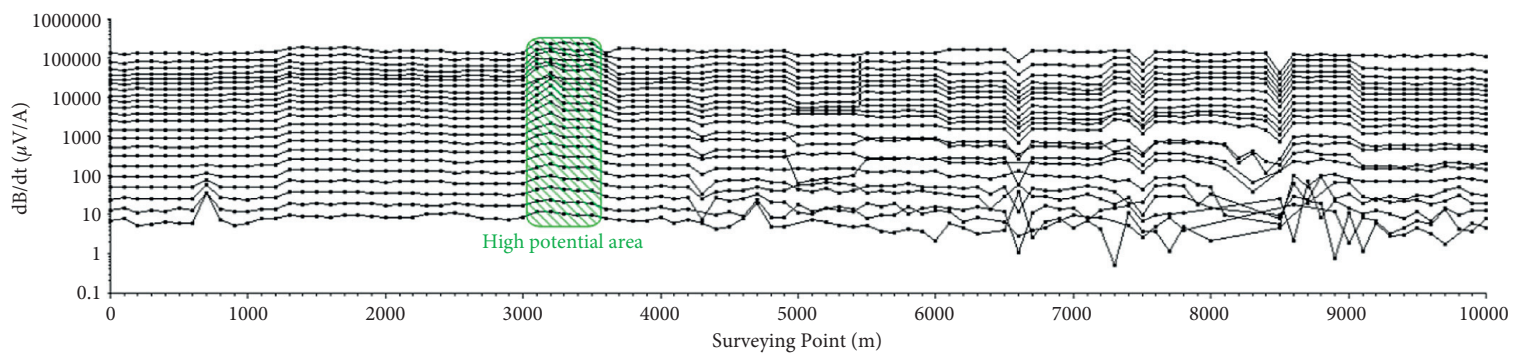

(b)

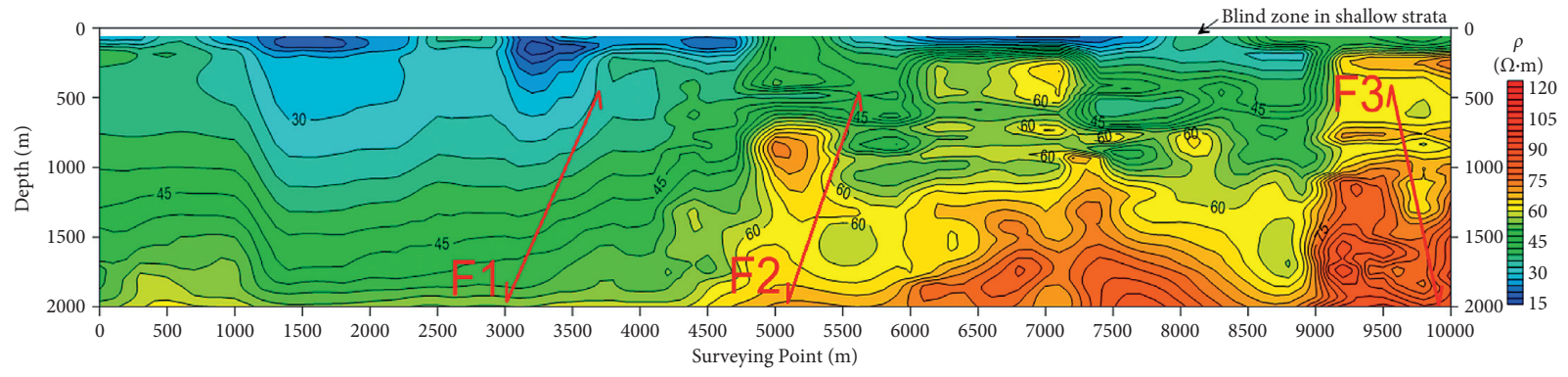

(c)

FIGURE 6: Contrast of CSAMT and TEM profiles of Line 60: (a). CSAMT resistivity profile, where the blue contours represent low resistivity, whereas the red contours represent high resistivity. (b). TEM secondary field profile, where the marked green area is the high potential area. (c). TEM resistivity profile, where the blue contours represent low resistivity, whereas the red contours represent high resistivity.

4.2. Discussion. In a certain exploration area, there is great variability in the geological conditions and interference conditions. The application of a single geophysical method for exploration will inevitably be affected by factors such as topography, surface construction difficulty, shielding layer, noise current, and effective exploration depth.

Through theoretical analysis and engineering examples, it is shown that the two electromagnetic methods, CSAMT and TEM, can both be used to effectively explore geothermal resources from different angles, as follows.

CSAMT has a great exploration depth and insufficient resolution for shallow layers, whereas TEM has a relatively small exploration depth and a strong exploration effect on shallow layers. At the same time, CSAMT possesses stronger anti-interference ability than TEM. Comprehensive exploration is adopted in the section with strong electromagnetic interference signal, which can improve the accuracy of data collection. CSAMT has higher requirements for signal emission and data collection, whereas the data collection of TEM is relatively simple. Comprehensive exploration in a complex construction environment can reduce the impact caused by construction. CSAMT exhibits obvious response to high-resistivity bodies in deep basement formations and can be used to explore high-resistivity geological bodies such as magmatic rocks. TEM has a strong exploration effect on low-resistivity geological bodies, such as water-conducting fault structures, and can increase the exploration resolution in the vertical direction. In the end, a comprehensive method was adopted for exploration in this study, and it was shown that both high- and low-resistivity bodies can be effectively explored in the vertical direction.

In this study, the comprehensive geophysical method with CSAMT as the main method and TEM as the supplemental one was used to achieve relatively ideal results in exploring geothermal resources in the magmatic rock areas. Compared with other geophysical or geological methods [30,52-58], the comprehensive geophysical method possesses significant advantages: By using the comprehensive geophysical method, the various elements of the geothermal system can be displayed. In addition, both thermal cap in shallow and magmatic rocks as the thermal reservoir in deep can be clearly described, and the water-conducting fault as 
the migration channel of geothermal fluid can also be delineated in detail.

\section{Conclusion}

CSAMT and TEM are both effective methods for geothermal resource exploration. However, each method has its limitations and scope of applicability. It is not possible to use only a single method to complete all geological tasks. The comprehensive geophysical method is shown to have a more reliable effect.

It should be noted that comprehensive geophysical prospecting is not simply a combination and addition of single geophysical prospecting methods. Instead, the ideal method should be selected according to the principles and characteristics of each geophysical prospecting method based on the geological task and the geological conditions of the exploration area, so as to maximize the advantages of each method.

Through the methodology, characteristic analysis, and engineering examples of the two different methods, it is shown that CSAMT is superior to TEM in deep exploration and magmatic rock basement exploration, whereas TEM is superior to CSAMT in shallow exploration and waterconducting fault structure exploration. The comprehensive geophysical method for geothermal exploration overcomes the limitations of a single method and has the effect of mutual complementation and confirmation. [6]

\section{Data Availability}

The data that support the findings of this study are available from the corresponding author upon request.

\section{Conflicts of Interest}

The authors declare no potential conflicts of interest with respect to the research, authorship, or publication of this article.

\section{Acknowledgments}

This research was supported by the Comprehensive Investigation and Evaluation Project of Geothermal Resources in the Cuona-Chayu Area, Tibet (Grant No. DD20211548).

\section{References}

[1] S. B. Hu, L. J. He, and J. Y. Wang, "Heat flow in the continental area of China: a new data set," Earth and Planetary Science Letters, vol. 179, no. 2, pp. 407-419, 2011.

[2] L. Zhang, S. Chen, and C. Zhang, "Geothermal power generation in China: status and prospects," Energy Science \& Engineering, vol. 7, no. 5, pp. 1428-1450, 2019.

[3] B. Shi, Geothermal Resources and its Development, Utilization and Protection, Chemical Industry Press, Beijing, China, 2005.

[4] G. L. Wang, F. Zhang, and Z. Liu, "An analysis of present situation and prospects of geothermal energy development and utilization in the world," Acta Geoscientia Sinica, vol. 21, no. 2, pp. 134-139, 2000.
[5] L. Yang, "On deployment of high- temperature deep geothermal wells in typical areas with hot dry rock potential in China," Geology and Exploration, vol. 53, no. 2, pp. 355-360, 2017.

[6] S. Soengkono, M. P. Hochstein, I. E. M. Smith, and T. Itaya, "Geophysical evidence for widespread reversely magnetised pyroclastics in the western Taupo Volcanic Zone (New Zealand)," New Zealand Journal of Geology and Geophysics, vol. 35, no. 1, pp. 47-55, 1992.

[7] G. Y. Liu and C. J. Zhang, "The role of microgravity survey in geothermal exploration," Geophysical \& Geochemical Expioration, vol. 18, no. 6, pp. 468-472, 1994.

[8] G. H. Xu, Q. F. Yu, and X. C. Yuan, "A tentative discussion on the application of the deep geothermal exploration method in Beijing area," Geophysical \& Geochemical Expioration, vol. 31, no. 1, pp. 9-13, 2007.

[9] S. J. Guo, B. X. Li, X. B. Zhou et al., "Application of remote sensing and electric method in the convective geothermal exploration of Ganzihe Fault in Qinghai," Computing Techniques for Geophysical and Geochemical Exploration, vol. 34, no. 5, pp. 566-570, 2012.

[10] G. Munoz, "Exploring for geothermal resources with electromagnetic methods," Surveys in Geophysics, vol. 35, no. 1, pp. 101-122, 2004

[11] M. N. Yin, C. An, and Y. Jin, "The application of the integrated geophysical and geochemical methods to the geothermal exploration in a certain area of Inner Mongolia," Geophysical and Geochemical Exploration, vol. 31, no. 4, pp. 313-316, 2007.

[12] Q. Y. Di, R. Wang, K. Shi, and Y. Li, "Applications of CSAMT for deep geothermal exploration in high level ambient noises areas in Beijing," Journal of Engineering Geology, vol. 15, no. Suppl, pp. 203-207, 2008.

[13] V. Spichak and A. Manzella, "Electromagnetic sounding of geothermal zones," Journal of Applied Geophysics, vol. 68, no. 4, pp. 459-478, 2009.

[14] Y. Yang, J. Liu, W. Zhang, G. Gao, B. Li, and F. Zhang, "Applying comprehensive geophysical prospecting method in geothermal exploration in Tianjin area," Global Geology, vol. 28, no. 3, pp. 351-360, 2009.

[15] X. Zhao, Z. Zeng, Z. Wu, K. Wang, J. Li, and T. Xu, "Delineating the area of HDR in Songliao basin using geophysical methods," Progress in Geophysics, vol. 30, no. 6, pp. 28632869, 2015.

[16] E. Aboud, "Geothermal exploration methods," Advances in Geothermal Energy, InTech, Rijeka, Croatia, pp. 149-151, 2016.

[17] L. Rybach and L. Muffler, Geothermal Systems: Principles and Case Histories, p. 371, Wiley-Interscience, New York, NY, USA, 1981.

[18] A. Berktold, "Electromagnetic studies in geothermal regions," Geophysical Surveys, vol. 6.1, no. 2, pp. 173-200, 1983.

[19] E. Huenges, "Enhanced geothermal systems: review and status of research and development," Geothermal Power Generation, vol. 25, pp. 743-761, 2016.

[20] L. Pellerin, J. M. Johnston, and G. W. Hohmann, "A numerical evaluation of electromagnetic methods in geothermal exploration," Geophysics, vol. 61, no. 1, pp. 121-130, 1996.

[21] W. D. Stanley, "Geothermal systems, principles and case histories," Earth-Science Reviews, vol. 18, no. 1, pp. 91-92, 1982.

[22] F. George, T. G. Caldwell, and H. M. Bibby, "Tensor time domain electromagnetic resistivity measurements at Ngatamariki geothermal field, New Zealand," Journal of Volcanology and Geothermal Research, vol. 127, pp. 33-54, 2003. 
[23] R. M. Rahman, S. Ata, and G. J. Jameson, "The effect of flotation variables on the recovery of different particle size fractions in the froth and the pulp," International Journal of Mineral Processing, vol. 106-109, pp. 70-77, 2012.

[24] R. Smith, "Electromagnetic induction methods in mining geophysics from 2008 to 2012," Surveys in Geophysics, vol. 35, no. 1, pp. 123-156, 2014.

[25] D. Curewitz and J. A. Karson, "Structural settings of hydrothermal outflow: fracture permeability maintained by fault propagation and interaction," Journal of Volcanology and Geothermal Research, vol. 79, no. 3-4, pp. 149-168, 1997.

[26] H. Alçiçek, A. Bülbül, and M. C. Alçiçek, "Hydrogeochemistry of the thermal waters from the yenice geothermal field (denizli basin, southwestern anatolia, Turkey)," Journal of Volcanology and Geothermal Research, vol. 309, pp. 118-138, 2016.

[27] M. Zucchi, "Faults controlling geothermal fluid flow in low permeability rock volumes: an example from the exhumed geothermal system of Eastern Elba Island (Northern Tyrrhenian Sea, Italy)," Geothermics, vol. 85, Article ID 101765, 2020.

[28] J. V. Rowland and R. H. Sibson, "Structural controls on hydrothermal flow in a segmented rift system, taupo volcanic zone, New Zealand," Geofluids, vol. 4, no. 4, pp. 259-283, 2004.

[29] D. Liotta, G. Ruggieri, A. Brogi, P. Fulignati, A. Dini, and I. Nardini, "Migration of geothermal fluids in extensional terrains: the ore deposits of the Boccheggiano-Montieri area (southern Tuscany, Italy)," International Journal of Earth Sciences, vol. 99, no. 3, pp. 623-644, 2010.

[30] D. Liotta, "Fracture analysis hydrothermal mineralization and fluid pathways in the Neogene Geitafell central volcano: insights for the Krafla active geothermal system, Iceland," Journal of Volcanology and Geothermal Research, vol. 391, p. 23, 2020.

[31] A. Brogi, M. C. Aliek, D. Liotta, E. Capezzuoli, and P. F. Matera, "Step-over fault zones controlling geothermal fluid-flow and travertine formation (Denizli Basin, Turkey)," Geothermics, vol. 89, pp. 101-941, 2021.

[32] J. S. Caine, J. P. Evans, and C. B. Forster, "Fault zone architecture and permeability structure," Geology, vol. 24, no. 11, pp. 1025-1028, 1996.

[33] L. Smith, C. B. Forster, and J. P. Evans, "Interaction of fault zone, fluid flow and heat transfer at the basin scale," Hydrogeology of Permeability Environments, vol. 2, pp. 41-67, 1990.

[34] G. Q. Xue, W. Chen, N. N. Zhou, and H. Li, "Short-offset TEM technique with a grounded wire source for deep sounding," Chinese Journal of Geophysics, vol. 56, no. 1, pp. 255-261, 2013.

[35] H. Amir, Development of Novel Time-Domain Electromagnetic Methods for Offshore Groundwater Studies: A Data Application from Bat Yam, Universität zu Köln, Cologne (Köln), Germany, 2016.

[36] H. Li, "Progresses in the research of magmatic rocks," Geology and Exploration, vol. 47, no. 2, pp. 180-186, 2010.

[37] L. Zhang and L. Lin, "Application of CSAMT in geothermal exploration,” Mining Technology, vol. 4, pp. 128-129+133, 2012.

[38] W. Li and Z. Liao, "The application of TEM to geothermal exploration in tengchong," Geophysical and Geochemical Exploration, vol. 26, no. 5, pp. 368-371, 2002.

[39] G. Garyet and J. R. DeGroot, "Robust estimation of geomagnetic transfer functions," Geophysical Journal of the Royal Astronomical Society, vol. 87, pp. 173-194, 2006.
[40] J. Li, "Overview of the development of electrical exploration methods," Geophysical and Geochemical Exploration, vol. 8, pp. 42-46, 1996.

[41] J. Wu, Y. Yang, X. Wang, J. Zhang, and X. Deng, “Application of TEM in the tianyu Cu-Ni deposit in East tian Shan, Xinjiang," Geology and Exploration, vol. 51, no. 5, pp. 970976, 2015

[42] H. Li, G.-Q. Xue, P. Zhao, N.-N. Zhou, and H.-S. Zhong, "Inversion of arbitrary segmented loop source TEM data over a layered earth," Journal of Applied Geophysics, vol. 128, pp. 87-95, 2016.

[43] H. Li, G. Q. Xue, N. N. Zhou, and W. Chen, "Appraisal of an array tem method in detecting a mined-out area beneath a conductive layer," Pure and Applied Geophysics, vol. 172, no. 10, pp. 917-2929, 2015.

[44] M. Yu, "Application of the transient electromagnetic (TEM) technology to the Keketale $\mathrm{Pb}-\mathrm{Zn}$ deposit in Xinjiang," $\mathrm{Ge}$ ology and Exploration, vol. 49, no. 4, pp. 723-730, 2013.

[45] J. Xu, A. Li, and S. Yang, "Calculation of transient magnetic field and all time apparent resistivity based on central TEM loops method," Geology and Exploration, vol. 44, no. 6, pp. 62-68, 2006.

[46] H. Li, Q. Y. Di, and G. Q. Xue, “A comparative study of inline and broadside time domain CSEM methods for mapping resistive targets on land," Geophysics, vol. 84, no. 4, pp. B235-B246, 2020.

[47] G. Q. Xue and J. C. Yu, "New development of TEM research and application in coal mine exploration," Progress in Geophysics, vol. 32, no. 1, pp. 319-326, 2017.

[48] H. Li, G. Q. Xue, and L. Zhang, "Accelerated Bayesian inversion of transient electromagnetic data using MCMC subposteriors," IEEE Transactions on Geoscience and Remote Sensing, vol. 59, no. 12, pp. 10000-10010, 2020.

[49] G. Q. Xue, "The discussion on the exploration depth of transient electromagnetic method: oil," Geophysical Prospecting, vol. 5, pp. 575-578, 2004.

[50] G. Q. Xue, J. Song, and S. Yan, "Analysis and estimation of minimum exploration depth for large loop transient electromagnetic method," Geotechnical Investigation \& Surveying, vol. 2, pp. 63-65, 2004.

[51] H. Li, G.-q. Xue, and P. Zhao, "A new imaging approach for dipole-dipole time-domain electromagnetic data based on the q-transform," Pure and Applied Geophysics, vol. 174, no. 10, pp. 3939-3953, 2017.

[52] G. Gola, G. Bertini, M. Bonini et al., "Data integration and conceptual modelling of the Larderello geothermal area, Italy,” Energy Procedia, vol. 125, pp. 300-309, 2017.

[53] J. Vidal and A. Genter, "Overview of naturally permeable fractured reservoirs in the central and southern Upper Rhine Graben: insights from geothermal wells," Geothermics, vol. 74, pp. 57-73, 2018.

[54] D. Liotta, A. Brogi, G. Ruggieri, and M. Zucchi, "Fossil vs. Active geothermal systems: a field and laboratory method to disclose the relationships between geothermal fluid flow and geological structures at depth," Energies, vol. 14, no. 4, p. 933, 2021.

[55] J. M. Millett, P. Sverre, K. Felix, B. Anett, P. H. Gylfi, and H. Sæunn, "Sub-surface geology and velocity structure of the Krafla high temperature geothermal field, Iceland: integrated ditch cuttings, wireline and zero offset vertical seismic profile analysis," Journal of Volcanology and Geothermal Research, vol. 391, pp. 106-342, 2020.

[56] E. Olvera-García, B. Caterina, V. H. Garduo-Monroy, B. Andrea, and L. Domenico, "Geological map of las minas: an 
example of an exhumed geothermal system (Eastern transMexican volcanic belt)," Journal of Maps, vol. 16, pp. 918-926, 2020.

[57] J. Klee, A. Chabani, B. A. Ledésert, S. Potel, R. L. Hébert, and G. Trullenque, "Fluid-rock interactions in a paleo-geothermal reservoir (Noble Hills Granite, California, USA). part 2: the influence of fracturing on granite alteration processes and fluid circulation at low to moderate regional strain," Geosciences, vol. 11, no. 11, p. 433, 2021.

[58] F. J. Guerrero, S. C. Giovanni, and M. P. Rosa, "A numerical model for the magmatic heat reservoir of the Las Tres Virgenes volcanic complex, Baja California Sur, Mexico," Journal of Volcanology and Geothermal Research, vol. 414, pp. 107227, 2021. 